\title{
Slow-Light Enhanced Second-Harmonic Generation Using a $\pi$-Phase Shifted Moiré Grating in a Quasi-Phased-Matched Medium
}

\author{
Thomas E Maybour, Devin H Smith, Peter Horak \\ Optoelectronics Research Centre, University of Southampton, Southampton SO17 1BJ, UK
}

Slow light is the phenomenon of a large reduction in group velocity, normally due to a material resonance [1]. Slow light causes spatial compression and consequently large field enhancement which has been suggested as a method for increasing nonlinear optical effects. Here we investigate the use of a Moiré grating [2,3] for enhancing second-harmonic generation (SHG) in a $\chi^{(2)}$ medium.

A Moiré grating is a slow-light device created by superimposing two Bragg gratings, leading to a doublebandgap structure separated by a slow-light transmission window. The grating has a superstructure period $\Lambda_{\mathrm{s}}$, larger values of which produce a smaller transmission band and lower group velocities. SHG also requires phase matching between the fundamental and the second harmonic mode, which in a nonlinear crystal can be achieved through quasi-phase matching (QPM) by periodic poling of the $\chi^{(2)}$ tensor [4] with period $\Lambda$ as illustrated in Fig. 1(a). Enhancement of SHG by slow light thus requires the combination of both QPM and a Moiré grating.

Our theoretical model of such a device is based on coupled mode equations (CMEs). The refractive index profile of the Moiré grating is $n(z)=\bar{n}+\delta n \operatorname{sgn}\left[\cos \left(2 \pi z / \Lambda_{s}\right)\right] \cos \left(2 \pi z / \Lambda_{B}\right)$, where $\bar{n}$ is the effective refractive index, $\delta n$ is the grating strength, and $\Lambda_{B}$ is the Bragg period. An example of the grating profile is shown in Fig. 1(a). The $\operatorname{sgn}\left[\cos \left(2 \pi z / \Lambda_{\mathrm{s}}\right)\right]$ term introduces periodic $\pi$-phase shifts into a regular Bragg grating, creating a transmission window. The CMEs for the fundamental (second harmonic) forward mode $u_{1}\left(u_{2}\right)$ and backward mode $v_{1}\left(v_{2}\right)$ are

$$
\begin{aligned}
& \frac{\partial u_{1}}{\partial z}=i \kappa_{1} f_{1}(z) \operatorname{sgn}\left[\cos \left(2 \pi z / \Lambda_{s}\right)\right] v_{1}-\operatorname{sgn}[\sin (\Delta \beta z)] \frac{i \chi^{(2)} \beta_{1}}{n_{1}^{2}} u_{1}^{*} u_{2} e^{i \Delta \beta z}, \\
& \frac{\partial v_{1}}{\partial z}=-i \kappa_{1} f_{1}(z) \operatorname{sgn}\left[\cos \left(2 \pi z / \Lambda_{s}\right)\right] u_{1}+\operatorname{sgn}[\sin (\Delta \beta z)] \frac{i \chi^{(2)} \beta_{1}}{n_{1}^{2}} v_{1}^{*} v_{2} e^{-i \Delta \beta z}, \\
& \frac{\partial u_{2}}{\partial z}=i \kappa_{2} f_{2}(z) v_{2}-\operatorname{sgn}[\sin (\Delta \beta z)] \frac{i \chi^{(2)} \beta_{2}}{n_{2}^{2}} u_{1}^{2} e^{-i \Delta \beta z}, \frac{\partial v_{2}}{\partial z}=-i \kappa_{2} f_{2}(z) v_{2}+\operatorname{sgn}[\sin (\Delta \beta z)] \frac{i \chi^{(2)} \beta_{2}}{n_{2}^{2}} v_{1}^{2} e^{i \Delta \beta z}
\end{aligned}
$$

where the Moiré grating coupling strength is given by $\kappa_{1}$ and the phase mismatch between fundamental and second harmonic is $\Delta \beta$. A second simple Bragg grating with coupling $\kappa_{2}$ is applied to the start of the crystal to filter the backward-going second harmonic mode to ensure the output is in the forward direction.

We demonstrate enhanced SHG by performing a simulation with an input pump intensity of $100 \mathrm{~kW} / \mathrm{cm}^{2}$ at $1550 \mathrm{~nm}$ in a $20 \mathrm{~mm} \mathrm{LiNbO}_{3}$ crystal with a nonlinear coefficient $\chi^{(2)}=25 \times 10^{-12} \mathrm{~V} / \mathrm{m}$ and a grating strength $\delta n=10^{-3}$. The Bragg period is set to $\Lambda_{B}=350.503 \mathrm{~nm}$ so that the pump is tuned to the centre of the Moiré transmission window. Fig. 1(b) shows the proportion of power in each of the modes exiting the crystal against the Moiré period. As the Moiré period is increased the proportion of power in the forward second harmonic mode increases until it reaches a maximum, after which the power is transferred into the backward fundamental mode. Fig. 1(c) compares the SHG along the grating in a standard QPM crystal with that enhanced by the optimised $\pi$ phase shifted Moiré grating $\left(\Lambda_{s}=5.39 \mathrm{~mm}\right.$, cf. Fig. 1(b)) demonstrating an enhancement of nonlinear power conversion by a factor of approximately 6 . For these parameters, the group velocity slow-down factor for the fundamental mode due to the Moiré grating, and thus the field enhancement factor, is 6.95.

In conclusion, we have demonstrated that applying a $\pi$-phase shifted Moiré grating to a QPM medium is an effective means of enhancing second-harmonic generation.

(a)

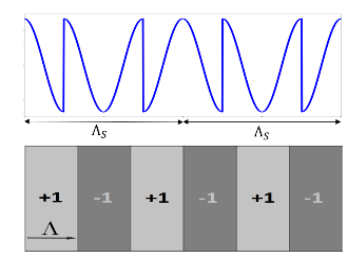

(b)

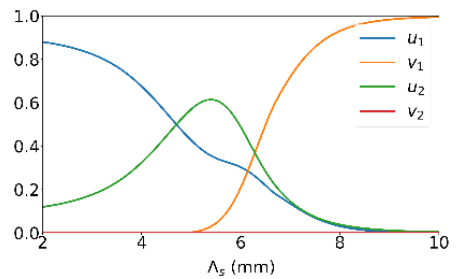

(c)

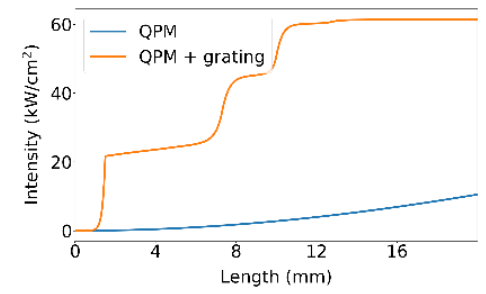

Fig. 1. (a) Schematic of a $\pi$-Phase Shifted Moiré Grating and $\chi^{(2)}$ QPM grating. (b) Proportion of power in the forward and backward fundamental $\left(\mathrm{u}_{1}, \mathrm{v}_{1}\right)$ and second harmonic $\left(\mathrm{u}_{2}, \mathrm{v}_{2}\right)$ modes at the outputs of the grating vs Moire grating period. (c) Comparison of SHG in a QPM crystal with and without slow-light enhancement by a Moiré grating.

[1] R. Won, "Slow light now and then," Nat. Photon. 2, 454 (2008).

[2] D. Janner et al., "Slow light in periodic superstructure Bragg gratings," Phys. Rev. E. 72, 056605 (2005).

[3] J. B. Khurgin, "Light slowing down in Moiré fiber gratings and its implications for nonlinear optics," Phys. Rev. A. 62, 013821 (2000).

[4] M. M. Fejer et al., "Quasi-phase-matched second harmonic generation: tuning and tolerances,” IEEE J. Quant. Electron. 28, 2631 (1992). 